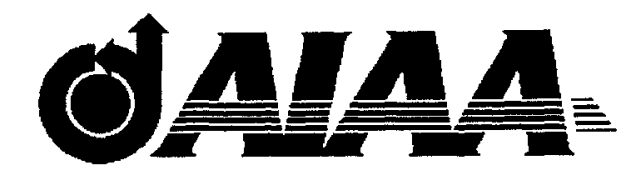

\title{
A01-40205
}

AIAA 2001-4527

\section{Autonomous Satellite Docking System}

P. Tchoryk, A. Hays, J. Pavlich, G. Wassick, G. Ritter, C. Nardell, G.

Sypitkowski

Michigan Aerospace Corporation

Ann Arbor, Ml

\section{Space 2001 Conference and Exposition 28-30 August 2001 Albuquerque, New Mexico}

For permission to copy or to republish, contact the copyright owner named on the first page. For AIAA-held copyright, write to AIAA Permissions Department, 1801 Alexander Bell Drive, Suite 500, Reston, VA, 20191-4344. 


\section{AUTONOMOUS SATELLITE DOCKING SYSTEM}

Pete Tchoryk, Jr." ${ }^{*}$, Anthony Hays ${ }^{\mathrm{a}}$, Jane Pavlich ${ }^{\mathrm{a}}$, Greg Wassick ${ }^{\mathrm{a}}$, Greg Ritter ${ }^{\mathrm{a}}$, Carl Nardell ${ }^{\mathrm{a}}$, Greg Sypitkowski $^{\mathrm{a}}$

${ }^{a}$ Michigan Aerospace Corporation, 1050 Highland Dr., Suite E, Ann Arbor, MI $48108^{1}$

\begin{abstract}
Michigan Aerospace has developed an Autonomous Satellite Docking System (ASDS) design along with mission concepts for the Orbital Express program. The new design and mission concepts are based on analysis and technology assessments performed as part of a Defense Advanced Research Projects Agency (DARPA) Phase I SBIR, in the areas of docking mechanisms and servicing systems, Guidance, Navigation, and Control (GNC), rendezvous and docking sensors, and avionics. Considerable attention was given to identifying solutions or development paths for key technology areas. A solution to one of those technologies, the docking mechanism, fills an immediate need and has resulted in a design that meets the requirements for a near-term onorbit demonstration. The docking mechanism was specifically designed for soft-docking capability and tolerance to misalignment. A servicing and re-supply system has also been designed around the docking mechanism, allowing multiple standard connections, such as fluid, electrical, and Orbital Replacement Unit (ORU) handling. This docking and servicing approach meets the needs of a near-term demonstration and provides a practical solution for the operational system. The docking and servicing system, as well as a new dynamic modeling and simulation capability, will be discussed in this paper.
\end{abstract}

\section{Introduction}

The cost of building and launching satellites limits the number of assets that can be placed in orbit. This makes it critical for the asset to have as long a lifetime as possible to reduce the replacement rate. The primary limiting factor of satellite on-orbit lifetime is its ability

\footnotetext{
* Principal Investigator and Director of Business Development.

${ }^{1}$ Copyright 2001 by the American Institute of Aeronautics and Astronautics, Inc. All rights reserved.
}

to maintain a useful, stable orbit. There is also a growing requirement to give satellites the ability to maneuver to new orbits on a regular basis. ${ }^{2}$ The ability of a satellite to change orbits on a regular basis makes it less vulnerable to attack, allows it to be repositioned to observe new crisis areas, and reduces the need for multiple-satellite constellations to provide global coverage. This comes down to the amount of fuel the satellite can carry to allow orbit maneuvers and pointing changes. Since the amount of fuel that a satellite can carry is limited by launch costs and weight limitations, the ability to re-fuel a satellite on-orbit is the only method available to increase its useful lifetime. Satellite lifetime is also determined by failures of critical components. If satellite architectures were designed for on-orbit servicing, it should also be possible to repair the satellite using an automated payload handling system to replace the damaged unit or replenish consumables (e.g., cryogenics, lasing gases, etc.)

\section{Heritage}

\section{COMET-BASED Autonomous Rendezvous and Docking (ARD) Experiment}

In 1991, the Space Automation and Robotics Center (SpARC), a NASA Center for the Commercial Development of Space, initiated a flight demonstration of Autonomous Rendezvous and Docking (ARD) technology originally scheduled to take place in the $1994 / 1995$ timeframe. ${ }^{3}$ The primary purpose of the mission was to demonstrate the feasibility of consumable (e.g., fuel) resupply and payload exchange. ${ }^{4}$ The initial demonstration was to make use of two Commercial Experiment Transporter (COMET) Service Modules that were to be launched by the Conestoga , an expendable launch vehicle built by EER Systems. By maintaining the first COMET spacecraft in orbit, a rendezvous and docking was to be performed

\footnotetext{
2 "New Space Research on Darpa's Horizon," Aviation Week \& Space Technology, July 19, 1999, pg. 58.

3 The COMET-ARD mission referred to here was an experiment proposed by the Environmental Research Institute of Michigan (ERIM), Space Automation and Robotics Center (SpARC), a NASA Center for the Commercial Development of Space. The payload was originally intended to fly on the Commercial Experiment Transporter (COMET). Key personnel are now with Michigan Aerospace.
}

${ }^{4}$ Our partners and contributors in this program included Oceaneering, Booz-Allen-Hamilton, MOOG, and Rockwell. 
with a second COMET spacecraft to be launched 18 months later.

COMET was developed to provide commercially available transportation and recovery services to the space experiment community. The service module was to remain on-orbit, acting as the target spacecraft, in anticipation of the docking with COMET 2, the chaser spacecraft. Although not specifically designed for $A R D$, the first COMET service module would have been made into a suitable target vehicle by retro-fitting with the SpARC docking receptacle, sensors, and avionics as pictured in Figure 1. The first service module was three-axis stabilized, but no orbit change capability existed. The second spacecraft was to contain the orbit adjust and maneuvering systems to enable rendezvous and docking.

In order for the docking to take place, however, the target spacecraft would have had to stay in orbit for approximately 18 months. As launch approached, it became apparent that the Conestoga launch vehicle could not meet its promised insertion altitude. COMET 1 would not be able to achieve the altitude required to survive until the next spacecraft arrived. Because of this, the ARD payload was pulled from the manifest. ${ }^{5}$

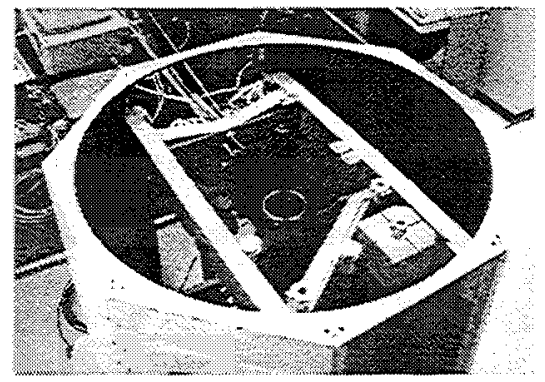

Figure 1. Top View of Docking Structure installed in COMET Service Module. ARD target payload integrated with the COMET Service Module at Space Industries (the docking interface is on the upper structure and the payload electronics are on the lower shelf)

5 The Conestoga carrying the first COMET spacecraft was launched from Wallops Island but had to be destroyed soon after launch because of a failure in one of the steerable rocket nozzles.
The COMET-based ARD demonstration was to be performed autonomously, but allowed for "man-in-theloop" intervention at certain stages along the way. By reducing the scope of the experiment to docking with a stable, cooperative target spacecraft, and taking advantage of the GPS system, the complexity and cost of validating ARD technology was considerably reduced.

\section{Phase I DARPA SBIR}

Because of the extensive amount of previous work performed in autonomous rendezvous and docking by the key personnel, the approach taken for the DARPA SBIR was to build from the existing system design. Changes in technology were assessed to determine an upgrade path that would lead to an on-orbit demonstration of the technology, similar to the COMET-ARD mission.

Particular emphasis was placed on evaluating docking mechanisms, rendezvous and docking sensors, and Guidance, Navigation \& Control (GN\&C) subsystems. All of the components for an on-orbit demonstration, however, were addressed as part of the Phase I SBIR. An evaluation of the state-of-the-art in technology for autonomous satellite docking and servicing was performed and data on the capabilities of new technologies and their application to autonomous operations were analyzed. Components that have recently been flown and tested in space, such as rendezvous and docking sensors and autonomous orbit control software were also reviewed. A baseline for an on-orbit demonstration had been established from the earlier COMET mission. Phase I was used to upgrade the existing system design to incorporate improvements in technology that had taken place over the past decade as well as update the docking scenario, mission planning and analysis, and performance estimates to reflect these technology improvements.

\section{Orbital Express Mission Concepts}

Orbital Express is a DARPA program that has the objective of demonstrating on-orbit autonomous satellite docking and servicing which would lead to the creation of an operational system to routinely refuel and service satellites. The on-orbit demonstration is intended to validate the technology and logistics of performing autonomous satellite servicing in LEO. Ultimately, the operational system must be capable of 
operating outside of LEO, to GEO and LaGrangian Points, as well.

\section{LEO Demonstration Concept}

A rendezvous and docking scenario and a firstlevel mission analysis were performed for a possible LEO demonstration based on the ASDS concept developed under the DARPA Phase I SBIR. As shown in Figures 2 and 3, a conceptual design of the docking system has been integrated with two spacecraft that could be used for the Orbital Express demonstration.

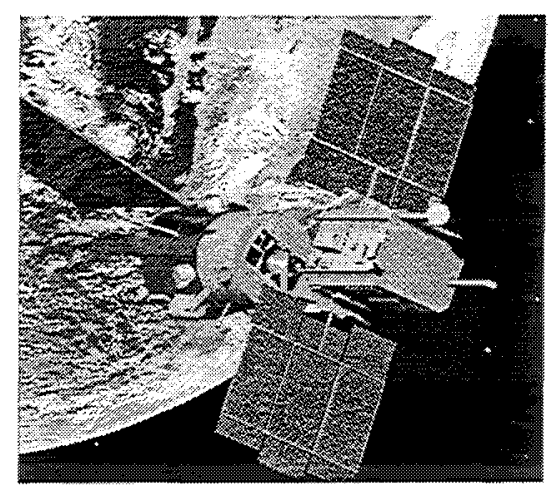

Figure 2. Rendezvous and Docking Model (Cone View)

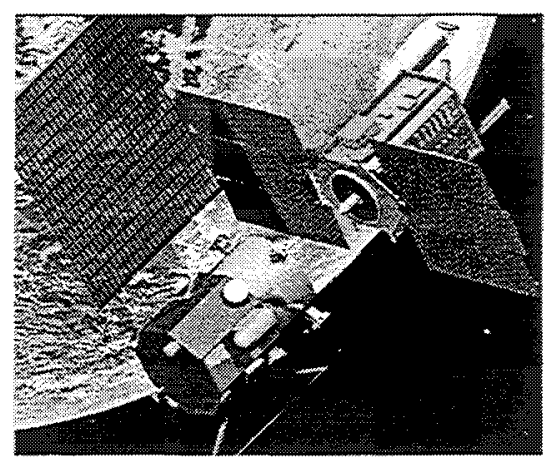

Figure 3. Rendezvous and Docking Model (Probe View)

\section{Mission Stages}

The mission stages referred to in this scenario are as follows:

\section{Rendezvous Stage}

a. Phasing (insertion orbit to $40 \mathrm{~km}$ )

2. Proximity ( $40 \mathrm{~km}$ to $100 \mathrm{~m})$

3. Terminal ( $100 \mathrm{~m}$ to Docking Stage)

4. Docking Stage ( $1 \mathrm{~m}$ to Mechanical Capture)

a. Soft-Dock (Capture/Latch)

b. Hard-Dock/Rigidization of the two spacecraft

\section{Docking and Servicing Mechanism Design}

One of the goals of the COMET-ARD mission a decade ago was to use as many pre-existing (off-theshelf) components as possible and to design new components only when absolutely necessary. Docking systems were investigated in great detail for COMET$\mathrm{ARD}$ and none were found to meet the requirements of compliance, size, weight and automation. A probe/cone docking system was designed specifically to allow the maximum compliance for autonomous operations while providing maximum flexibility for servicing functions.

Docking mechanism technology was again reviewed and trade studies performed as part of the DARPA Phase I SBIR. As a result of the technology assessment, it was determined that the probe/cone/flexible-cable docking mechanism design provides the most viable solution for both a near-term on-orbit demonstration as well as the future operational system.

Several new design concepts based on the probe/cone approach were evaluated. The current design allows a high degree of tolerance for aligning connectors and ports. A servicing mechanism concept was then designed that took advantage of the compact, compliant nature of the docking mechanism. The servicing mechanism was designed to provide maximum flexibility in terms of the types of connection that were supported, while minimizing the complexity of the connection process. This approach utilizes a simple, mechanized method for ORU transfer. Fluid/electrical/thermal ports can easily be accommodated around the periphery of the docking mechanism, providing flexibility in the number and type of connectors.

The following docking sequence is graphically illustrated in Figure 4:

1. STATION-KEEPING: Using inputs from the docking sensor, the AGNC guides the chase vehicle to a station-keeping position, maintaining approximately $1 \mathrm{~m}$ separation between the two spacecraft. 
2. SOFT-DOCKING: The soft-dock operation commences when the flexible cable is extended toward the target vehicle. At approximately $1 / 2 \mathrm{~m}$ separation, the boom is extended until the cable latches at the bottom of the cone on the target vehicle. The two spacecraft are now soft-docked, with only minimal forces having been applied to the target spacecraft.

3. HARD-DOCKING: Once the probe is latched in the cone, the cable retracts, pulling the boom into contact with the cone. When the probe head is fully seated within the cone, the two spacecraft are hard docked.

4. RIGIDIZATION: At this point, the boom is then retracted to bring the stabilizer probes (used for larger spacecraft) into contact with the target vehicle. Roll alignment takes place passively as the stabilizer probes are guided into place (or actively by rotating the probe head). Once the stabilizer probes are engaged in their own miniature versions of the main docking cone, the main boom pushes forward to put tension on the stabilizers, providing a rigid connection.

5. SERVICING: The two spacecraft are now rigidly docked and aligned closely enough to allow fluid and electrical connections to be mated with the target vehicle.
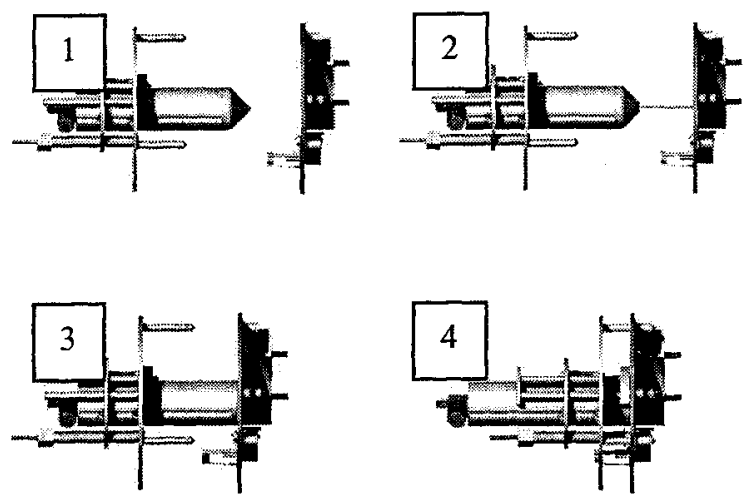

Figure 4. ASDS Docking Sequence; (I) Probe at station-keeping distance, (2) Flexible cable extended to perform soft-dock operation, (3) Cable retracted to perform hard-dock operation (final rotational alignment can be performed passively using the stabilizer posts or actively by rotating the probe head),

(4) Probe retracted to perform final rigidization and allow servicing.

Figures 5 and 6 contain the passive (cone) side and active (probe) side, respectively, of the docking and servicing interface.

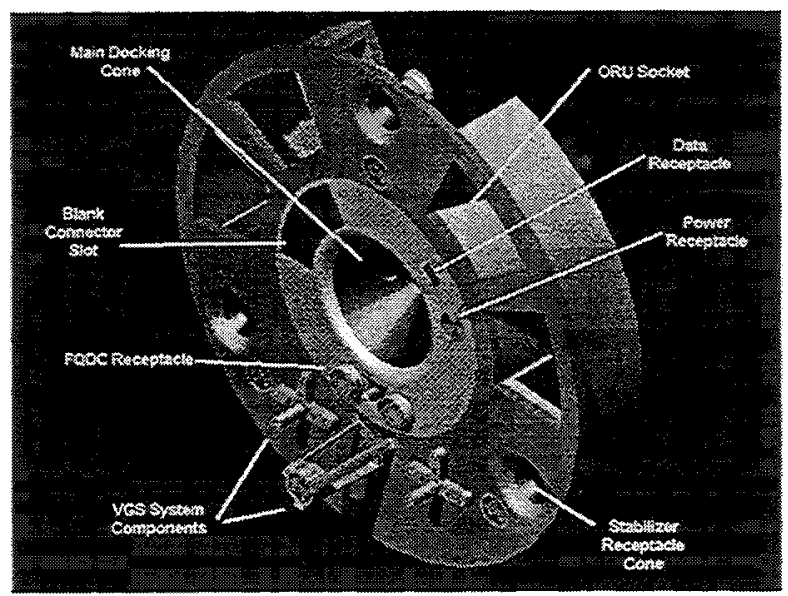

Figure 5. Passive (Cone) Side of the Docking and Servicing Interface

4

American Institute of Aeronautics and Astronautics 


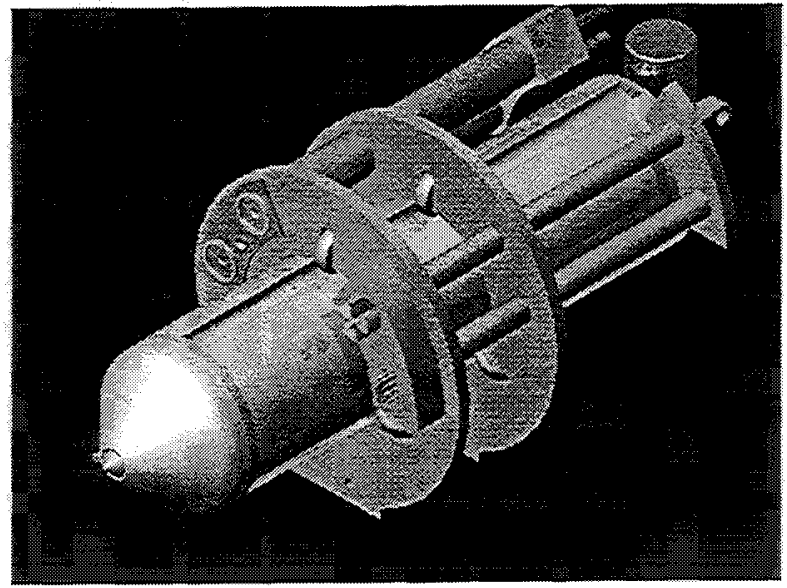

Figure 6. Active (Probe) Side of the Docking and Servicing Interface

The servicing concept shown in the figures above uses the boom facing plate to hold the fluid connectors (left hand side) and electrical and power connectors (right hand side). The blue canister toward the back of the unit is the linear actuator motor that drives the cable. This view of the docking system shows the main docking boom without stabilizers, which are unnecessary for smaller target vehicles. The same docking probe can be used to dock with and service any sized target satellite with this concept. The number and type of connectors and rigidization points may differ between satellites, depending upon their size and function, but the same servicing vehicle can still service any satellite.

In the case of larger satellites, three rigid stabilizer probes can be added; each of which hold a mechanism similar to the cable-mounted soft-dock mechanism. In this concept, the stabilizer probes engage in miniature versions of the main docking cone and provide a rigid connection between the chaser and target vehicles.

\section{Dynamic Modeling and Simulation}

One of the goals of this program was to create a dynamic model of the entire rendezvous and docking process. The model incorporates empirical "realworld" data, as well as theoretical models of probe/cone performance. Simulations can be performed using solid models of various docking mechanism designs, providing enhanced visualization of docking scenarios. Parameters and components of the probe/cone mechanism can then be modified to analyze the performance of the mechanism under various spacecraft configurations and mission scenarios.

The basis for the dynamic model of the docking mechanism is provided by Mechanical Dynamics' ADAMS software. In the current model, many different aspects of the docking process can be analyzed, including the following:

- Tip-off forces acting on target cone (Soft Dock)

- Cable Retraction Force

- Forces acting on docking system during initial probe/cone contact

- Forces acting on cable and boom structure during initial contact

- Flexure of boom structure under transverse loading

- Forces acting on cable and boom structure during auto alignment and rigidization

- Docking cable oscillation under stationkeeping conditions

- The frequency of oscillation of the cable

- The stiffness required of the cable to prevent oscillation

The intent of this model is to be able to examine a number of docking scenarios between a chaser and target satellite. of specific interest is the behavior/loading of the cable/latch subassembly used to capture, position and dock the target satellite. It is desired to vary model parameters such as the initial satellite relative positions, their masses, the cable/latch stiffness, and cable/probe deployment speeds.

The model consists of a target vehicle located at a (user-specified) stand-off distance and orientation with respect to the chaser vehicle, a simplified model of which is shown in Figure 7. Contact modeling represents one of the main elements in this analysis. To accomplish this, the new, ADAMS Version 11, Parasolids-based, 3-D CONTACT has been employed. Results of this analysis and modeling will be reported at a later date. Ultimately, the model will incorporate algorithms for orbit transfer, orbit control, proximity operations and docking, allowing full end-to-end simulation of the docking process. 

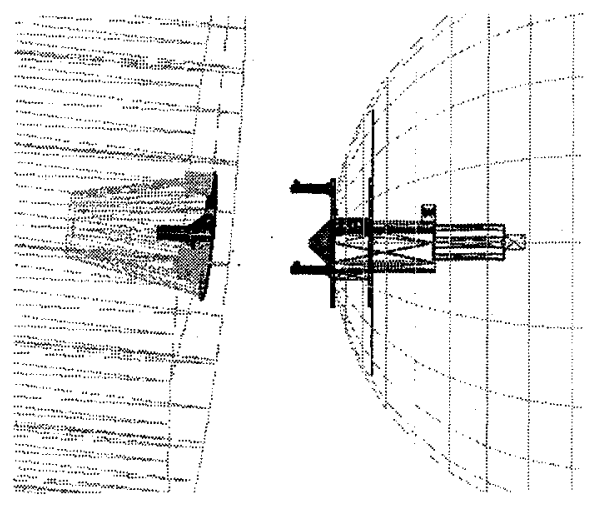

Figure 7. ADAMS Model of Docking Mechanism

\section{Conclusions}

Analysis and technology assessments were performed in the areas of docking mechanisms, servicing systems, Guidance, Navigation, and Control (GNC), rendezvous and docking sensors, and avionics. Based on this analysis, several key technology areas were identified that required development and/or testing to meet Orbital Express autonomous satellite docking needs.

A solution to one of those key requirements is the Michigan Aerospace docking mechanism. A significant portion of the development work in the Phase I SBIR was directed toward the probe/cone docking mechanism. With this concept, a single docking and servicing system on-board a servicing spacecraft can dock with and service any target satellite.

The technology development and testing plan proposed by Michigan Aerospace will help meet the requirements of the Orbital Express on-orbit demonstration and promote the maturation of technology required for the operational system. Laboratory testing of the current prototype is underway and will be reported on at a later date, along with complete performance specifications.

Other applications for autonomous rendezvous and docking are also being investigated at this time, including the following:

- Mars Sample Return scenarios

- Microsatellite operations

- Alternate Access to Station.
Acknowledgements

The authors and Michigan Aerospace Corporation would like to express their sincere appreciation to the sponsors of this research and development, Major Jim Shoemaker and Mr. Sam Wilson, DARPA Program Managers. The authors would also like to thank Microcosm and Mechanical Dynamics (in particular, Jim McConville) for their continued support in this area.

\section{Bibliography}

1. "Autonomous Rendezvous and Docking Scenarios for Guidelines and Standards," Quintero, Montgomery, Tchoryk, AIAA Space Programs Conference Proceedings, Huntsville, AL, September 21-23, 1993.

2. "Autonomous Rendezvous and Docking Using an Expendable Launch Vehicle," Tchoryk, Dobbs, Apley, Conrad, Frazer, AIAA/Utah State University Conference on Small Satellites, Poster Paper, Logan, Utah, August 27-29, 1991.

3. "Passive and Active Sensors for Autonomous Space Applications," Tchoryk, Gleichman, Carmer, Morita, Trichel, Gilbert, SPIE Aerospace Sensors Symposium, Surveillance Technologies Conference, Orlando, FL, Vol, 1479, pp. 164-182, April 2-5, 1991.

4. "Application of Laser Radar to Autonomous Spacecraft Landing," Gleichman, Tchoryk, Sampson, SPIE International Symposium on High-Power Lasers, Laser Radar VI Conference, Los Angeles, CA, January 20-25, 1991.

5. "Sensor Trade Study Volume 1: Autonomous Rendezvous and Docking," Gilbert, Carmer, Gleichman, Morita, Tchoryk, ERIM Report 216900-8-F1, Environmental Research Institute of Michigan, Ann Arbor, Ml, June 1990.

6. "Technology of Automated Rendezvous and Capture in Space," M.E. Polites, Journal of Spacecraft and Robotics, Volume 36, Number 2, pp. 280-291, March - April, 1999.

7. On-Orbit Servicing of Space Systems, D.M. Waltz, Krieger Publishing Co., Malabar, FL, 1993. 
8. Spacecraft Structures and Mechanisms From

Concept to Launch, T.P. Sarafin, ed.,

Microcosm Press, Torrance, CA, 1998.

9. Space Vehicle Mechanisms, P.L. Conley, ed., John Wiley \& Sons, Inc., New York, 1998.

10. Principles of Space Instrument Design, A.M Cruise, J.A. Bowles, T.J. Patrick, and C.V. Goodall, Cambridge University Press, Cambridge, United Kingdom, 1998.

11. Space Mission Analysis and Design, J.R. Wertz and W.J. Larson, ed., $3^{\text {rd }}$ Edition, Microcosm Press, Torrance, CA, 1999. 